\title{
Importância do tecido ceratinizado para o sucesso na implantodontia
}

\author{
Importance of keratinized tissue in implantology success \\ Importancia del tejido queratinizado para el éxito em implantología
}

Recebido: 20/01/2021 | Revisado: 23/01/2021 | Aceito: 25/01/2021 | Publicado: 03/02/2021

Thaís Kazue Nagai
ORCID: https://orcid.org/0000-0002-2361-4852
Universidade do Oeste Paulista, Brasil
E-mail: thaisnagai@ hotmail.com
Anderson Maikon de Souza Santos
ORCID: https://orcid.org/0000-0001-9371-9417
Universidade Estadual Paulista, Brasil
E-mail: andersonmaikon@ @otmail.com
Nathália Evelyn da Silva Machado
ORCID: https://orcid.org/0000-0002-4196-782X
Universidade Estadual Paulista, Brasil
E-mail: nathalia.machado@ @nesp.br
Bruno Coelho Mendes
ORCID: https://orcid.org/0000-0001-7896-8909
Universidade Estadual Paulista, Brasil
E-mail: brunocoelho.mnds@ gmail.com
Tiburtino José de Lima Neto
ORCID: https://orcid.org/0000-0002-8297-4057
Universidade Estadual Paulista, Brasil
E-mail: tiburtinoneto@ hotmail.com
Ana Maria Veiga Vasques
ORCID: https://orcid.org/0000-0002-1211-2363
Universidade Estadual Paulista, Brasil
E-mail: anavvasques@ @otmail.com
Eloi Dezan Júnior
ORation
ORCID: https://orcid.org/0000-0002-5699-6578
Universidade Estadual Paulista, Brasil
E-mail: eloi.dezan@ @unesp.br
Leonardo Perez Faverani
Universidade Estadual Paulista, Brasil
E-mail: leonardo.faverani@ unesp.br

\section{Resumo}

Fundamento: uma boa condição dos tecidos moles é necessária para a longevidade de tratamentos com implantes dentários. Os tecidos moles periimplantares são semelhantes ao periodonto de proteção, sendo importante para oferecer uma barreira contra a agressão bacteriana ao tecido ósseo. Objetivo: o objetivo foi realizar uma revisão de literatura sobre a importância da mucosa queratinizada na implantodontia; analisando a relação entre a manutenção gengival e saúde periimplantar. Metodologia: bases de dados foram acessadas para realização da pesquisa de artigos publicados na literatura Odontológica, na língua portuguesa e inglesa, principalmente entre os anos de 2010 a 2019. As bases de dados acessadas foram: LILACS, PUBMED, Scielo e a Biblioteca Digital Brasileira de Teses e Dissertações (BDTD). As palavras chaves usadas foram: osseointegration AND periodontics AND mouth mucosa AND gingiva. Discussão: vários estudos, desde estudos animais à revisões sistemáticas de literatura foram realizadas para avaliar o papel do tecido ceratinizado na implantodotia, e observaram que este tecido atua positivamente, com melhora do selamento periimplantar, redução de inflamação local, melhora na realização da higiene local e menos casos de recessão tecidual. Conclusão: conclui-se que o tecido ceratinizado é fundamental para a saúde periimplantar, atuando tanto na saúde oral do paciente reabilitado, quanto na sua estética e funcionalidade.

Palavras-chave: Osseointegração; Periodontia; Mucosa bucal; Gengiva.

\begin{abstract}
Introduction: a good condition of the soft tissues is necessary for the longevity of treatments with dental implants. The peri-implant soft tissues are similar to the protection periodontium, being important to offer a barrier against bacterial aggression to bone tissue. Objective: the objective was to conduct a literature review on the importance of the keratinized mucosa in implantology; analyzing the relationship between gingival maintenance and peri-implant health. Methodology: databases were accessed to conduct research on articles published in the Dental literature, in
\end{abstract}


Portuguese and English, mainly between the years 2010 to 2019. The databases accessed were: LILACS, PUBMED, Scielo and the Brazilian Digital Library Theses and Dissertations (BDTD). The keywords used were: osseointegration AND periodontics AND mouth mucosa AND gingiva. Discussion: several studies, from animal studies to systematic literature reviews, were carried out to evaluate the role of keratinized tissue in implantology, and observed that this tissue acts positively, with improvement of peri-implant sealing, reduction of local inflammation, improvement in the performance of local hygiene and less cases of tissue recession. Conclusion: it is concluded that the keratinized tissue is fundamental for periimplant health, acting both in the oral health of the rehabilited patient, as well as in its aesthetics and functionality.

Keywords: Osseointegration: Periodontics; Mouth mucosa; Gingiva.

\section{Resumen}

Antecedentes: un buen estado de los tejidos blandos es necesario para la longevidad de los tratamientos con implantes dentales. Los tejidos blandos periimplantarios son similares al periodonto de protección, siendo importante ofrecer una barrera contra la agresión bacteriana al tejido óseo. Objetivo: el objetivo fue realizar una revisión de la literatura sobre la importancia de la mucosa queratinizada en la implantología; analizar la relación entre el mantenimiento gingival y la salud periimplantaria. Metodología: se accedió a bases de datos para la búsqueda de artículos publicados en la literatura odontológica, en portugués e inglés, principalmente entre los años 2010 a 2019. Las bases de datos accedidas fueron: LILACS, PUBMED, Scielo y la Biblioteca Digital Brasileña Tesis y Disertaciones (BDTD). Las palabras clave utilizadas fueron: osteointegración Y periodoncia Y mucosa bucal Y encía. Discusión: se realizaron varios estudios, desde estudios en animales hasta revisiones sistemáticas de la literatura, para evaluar el papel del tejido queratinizado en implantología, y se observó que este tejido actúa positivamente, con mejora del sellado periimplantario, reducción de la inflamación local, mejora en el desempeño de la higiene local. y menos casos de recesión tisular. Conclusión: se concluye que el tejido queratinizado es fundamental para la salud periimplantaria, actuando tanto en la salud bucal del paciente rehabilitado, como en su estética y funcionalidad.

Palabras clave: Osteointegración: Periodoncia; Mucosa oral; Encía.

\section{Introdução}

No início da implantodontia, a pesquisa era focada principalmente na análise da integração do implante com o tecido ósseo. Porém, nos últimos anos os implantes dentários revolucionaram o procedimento de reabilitação oral, apresentando altas taxas de sucesso e muitas possibilidades de substituição dental (Lages, Douglas-de Oliveira, \& Costa, 2018). Os dentes possuem o ligamento periodontal, que é uma estrutura de tecido conjuntivo frouxo, ricamente celularizado e vascularizado, que se situa entre o cemento e o osso alveolar (Carranza, Newman, Takei, \& Klokkevold, 2011). Já os implantes possuem a osseointegração com o contato direto do osso com o implante sem camada intermediária de tecido mole, que é um dos parâmetros mais importantes avaliados em longo prazo ( Heinemann, Hasan, Bourauel, Biffar, \& Mundt, 2015).

Após a instalação dos implantes, ocorre uma sequência de eventos na interface osso/implante, que são relevantes para o sucesso do procedimento (Sciasci et al., 2018). Dentre esses eventos, nós podemos citar a estabilidade primária, que está associada ao embricamento mecânico do implante com o osso circundante no ato da instalação, (Javed, Ahmed, Crespi, \& Romanos, 2013) e posteriormente a estabilidade secundária, proporcionada pela deposição de osso recém-formado ao redor do corpo do implante (Silva et al., 2018). A qualidade desta estabilidade, no entanto, parece ser altamente dependente da estabilidade primária (Zita Gomes Vasconcelos, Lopes Guerra, de Almeida, \& de Campos Felino, 2017).

Nos últimos anos vem-se estudando mais a área periimplantar e as reações dos tecidos moles na implantodontia com uma maior ênfase, sendo de extrema importância garantir que os implantes, além de osseointegrados, mantenham seu estado de plena saúde, função e estética adequada, principalmente em relação à estética gengival (Garcia, Kraehenmann, Bezerra, Mendes, \& Rapp, 2008; Novaes, Santos, Almeida, Pellizer, \& Mendonça, 2012).

Ao redor de implantes dentários é comum não haver mucosa ceratinizada, pela inexistência de ligamento periodontal. Uma boa condição dos tecidos moles é necessária para a longevidade de tratamentos com implantes dentários. Os tecidos moles periimplantares são semelhantes ao periodonto de proteção, sendo importante para oferecer uma barreira contra a agressão bacteriana ao tecido ósseo. Algumas características importantes da mucosa ceratinizada, que oferecem uma maior 
proteção ao periodonto são a impermeabilidade e a imobilidade, que são conferidas a mucosa graças a camada de queratina e fibras colágenas respectivamente (Dinato \& Polido, 2004).

Atualmente com o aumento da preocupação com a estética nos tratamentos odontológicos, em relação aos tecidos moles e estética rosa, existe alguns cuidados e parâmetros a serem seguidos para que se chegue a um resultado reabilitador satisfatório tanto no ponto de vista funcional como estético. Alguns parâmetros que devem ser levados em conta nas fases de planejamento são: a criação de uma margem gengival harmônica, uma completa formação de papila interdental e o biótipo periodontal (Albrektsson Zarb, Worthington, \& Eriksson, 1986; Park et al., 2010; Soriní-Bröker, Peñarrocha-Diago, \& Peñarrocha-Diago, 2009).

Albrektsson et al. (1986), afirmaram que a presença suficiente de mucosa queratinizada ao redor de implantes permite que os pacientes mantenham uma boa higiene oral rotineira, sem irritação ou desconforto.

Saber identificar o biótipo periodontal antes do procedimento reabilitador é de suma importância, para que a manipulação do tecido seja adequada de acordo com a espessura do tecido, para que ao fim do tratamento as expectativas estéticas e funcionais sejam atendidas de forma satisfatória (Kao, Fagan, \& Conte, 2008). O fenótipo gengival pode influenciar diretamente na estética periimplantar, uma vez que um fenótipo fino tende a ser mais translucido, com pouca quantidade de gengiva inserida, tornando o prognóstico do tratamento, do ponto de vista estético, imprevisível se comparado com um fenótipo gengival espesso com maior quantidade de tecido queratinizado, que tende a resistir melhor às fases cirúrgicas que esse tratamento necessita (Adell et al., 1986; Brito, Tenenbaum, Wong, Schmitt, \& Nogueira-Filho et al., 2014; Branemark, Zarb, \& Albrektsson et al., 1985; Casado, Bonato \& Granjeiro, 2013; Chiu, Lee, Lin, \& Lai, 2015; Gallucci, Grütter, Chuang, \& Belser, 2011; Gobbato, Avila-Ortiz, Sohrabi, Wang, \& Karimbux, 2013; Lin, Chan, \& Wang, 2013; Novaes et al., 2012; Pranskunas, Poskevicius, Juodzbalys, Kubilius, \& Jimbo, 2016).

Se o cirurgião dentista estiver ciente destes fatores na fase de planejamento, ele pode minimizar uma eventual reabsorção óssea criando um ambiente mais favorável à instalação de implantes osseointegrados (Kao et al., 2008).

Quando da falta de uma faixa adequada de gengiva ceratinizada, podemos compensar com cirurgias mucogengivais, que são cirurgias que visam reestabelecer uma faixa adequada de mucosa ceratinizada, corrigindo as relações entre gengiva e mucosa. As cirurgias mucogengivais também auxiliam a melhorar a saúde periodontal, ajudando a interpor uma barreira às tensões geradas pelos freios e bridas (Bassetti, Stähli, Bassetti, \& Sculean, 2017; Luo, Chvartszaid, Kim, \& Portnof, 2020; Tavelli et al., 2020).

Desta forma, estudos que revisem a importância do tecido ceratinizado na implantodontia torna-se de extrema importância para auxiliar os cirurgiões dentistas na execução deles, além de trazer para o paciente uma maior satisfação pósoperatória quanto à higienização, a função gengival e a estética em casos de reabilitação protética sob implante ósseo.

O objetivo foi realizar uma revisão de literatura sobre a importância da mucosa queratinizada na implantodontia; analisando a relação entre a manutenção gengival e saúde periimplantar.

\section{Metodologia}

Foi executada uma revisão não sistemática no período compreendido entre 2010 a 2020, nas bases de dados vinculadas à Biblioteca Virtual da Saúde (BVS) - Bireme: LILACS, PUBMED, Scielo e a Biblioteca Digital Brasileira de Teses e Dissertações (BDTD), com a seguinte estratégia de busca: (dental implants AND periodontics AND mouth mucosa AND gingiva), sendo adaptada a cada duas palavras, depois três, e por fim, as quatro palavras chaves juntas para uma busca ampla sobre o assunto; além de ter buscado idiomas das diferentes bases de dados. A revisão realizada contou com a análise qualitativa, seguindo os padrões apresentados por Lin, et al., (2013). Na análise das publicações, as informações foram agrupadas de modo a estabelecer a importância da gengiva ceratinizada na reabilitação por implantes. 


\subsection{Revisão de literatura}

O sucesso da implantodontia é cada dia mais um desafio, e uma série de fatores está relacionada à saúde periimplantar, onde busca-se a harmonia entre os tecidos bucais (osso e tecido gengival) para que se obtenha o resultado esperado, tanto estético quanto funcional (Adell et al., 1986; Branemark al., 1985; Hammerle \& Tarnow, 2018; Marcantonio Nicoli, Marcantonio Junior, \& Zandim-Barcelos et al., 2015; Park et al., 2010; Tavelli et al., 2020).

Um dos fatores mais citados na literatura que se refere ao sucesso dos implantes dentários é a presença de uma faixa adequada de mucosa ceratinizada. Albrektsson et al, (1986) já concluíram em seus estudos que uma quantidade suficiente de tecido ceratinizado é necessário para reduzir a irritação e facilitar a higienização periimplantar.

Por este motivo, deve ser dada atenção para avaliação da quantidade e qualidade de tecido ceratinizado, bem como avaliação do biotipo gengival. Este pode ser classificado em biotipo fino e espesso, onde cada tipo reagirá diferentemente aos estímulos agressivos aplicados a estes (Kahn, Menezes, Imperial, Leite, \& Dias, 2013; Malpartida-Carrillo et al., 2020; Marcantonio et al., 2015). O biotipo fino geralmente reage aos traumas com recessão gengival enquanto que o biotipo espesso tende a formação de bolsa periodontal, mas com manutenção da altura tecidual (Kahn et al., 2013; Malpartida-Carrillo et al., 2020; Rebollal, Vidigal-Júnior. \& Cardoso, 2006). Além disso, o biotipo espesso, tem melhor proteção do tecido ósseo adjacente, apresentando menos reabsorção vestibular e interproximal. (Kahn et al., 2013; Malpartida-Carrillo et al., 2020; Rebollal et al., 2006). Diante disso, a avaliação do biotipo gengival é fundamental para determinar o planejamento reabilitador adequado, promovendo melhores resultados estéticos e funcionais.

Como já evidenciado por Casado et al, (2013) o biotipo gengival é um importante fator a ser observado quando se busca a saúde periimplantar, pois uma faixa adequada de tecido ceratinizado é primordial para o sucesso estético e para estabilidade do implante tardiamente.

Histologicamente, o tecido de gengiva ceratinizada é observada como uma faixa de epitélio escamoso estratificado ceratinizado e tecido conjuntivo denso inserido diretamente ao periósteo, enquanto o restante da mucosa bucal se apresenta com tecido conjuntivo frouxamente organizado e epitélio não ceratinizado (Listgarten, Lang, Schroeder, \& Schroeder, 1991).

A presença de faixa de mucosa ceratinizada adequada promove uma vedação biológica em torno do implante dentário, reduzindo a infiltração de microrganismos e facilitando a higienização da área, consequentemente diminuindo a inflamação tecidual local, que por sua vez atua no processo de reabsorção óssea e reduz o sucesso dos implantes (Marcantonio et al., 2015; Novaes et al., 2012).

Assim como observado nos dentes naturais, o biotipo gengival espesso apresenta menor índice de recessão gengival, mas com formação de bolsas periodontais, de maneira que a espessura da mucosa periimplantar atua diretamente na dinâmica entre os tecidos periimplantares (Zigdon \& Machtei, 2008). Além disso, no que se refere à estética periimplantar, o biotipo gengival fino, que está mais relacionado às recessões gengivais, acaba desenvolvendo maior número de casos com exposição de espiras dos implantes, comprometendo o sucesso estético, principalmente nas regiões anteriores. (Gallucci et al., 2011)

A reabsorção óssea também está mais relacionada aos implantes que são circundados por mucosa não ceratinizada. De acordo com Bouri et al (2008), o aumento da faixa de gengiva ceratinizada está diretamente relacionado a um maior sucesso dos implantes, onde estes apresentam saúde periimplantar ampliada.

Uma série de revisões sistemáticas (Brito et al., 2014; Chiu et al., 2015; Gobbato et al., 2013; Lin et al., 2013; Pranskunas et al., 2016) já tenham sido feitas acerca do tema e mostraram que, ao menos $2 \mathrm{~mm}$ de mucosa ceratinizada periimplantar é fundamental para o sucesso dos implantes dentários, pois reduzem o acúmulo de placa bacteriana, a inflamação tecidual, a recessão gengival e perda no selamento biológico. No entanto ainda são necessários novos estudos para compreender a relação entre a mucosa ceratinizada e alteração do nível osso periimplantar (Lin \& Madi, 2019). 
No entanto, é compreendido que é necessário 3mm para formação do o espaço biológico periimplantar, sendo assim, se não houver quantidade suficiente de tecido gengival, haverá uma reabsorção da crista óssea possibilitando assim, a formação deste espaço (Lin \& Madi, 2019).

Uma serie de técnicas cirúrgicas são descritas para adaptação quando a quantidade de mucosa ceratinizada ou o biotipo gengival são inadequados para o sucesso do implante dentário a longo prazo, entre estas técnicas, podemos citar reposicionamento gengival, enxertos livres de gengiva e enxertos de tecido conjuntivo como as técnicas com resultados mais satisfatórios, mas outras também há disponibilidade no mercado de matriz de colágeno xenógena (Bassetti et al., 2017; Luo et al., 2020; Tavelli et al., 2020). Essas técnicas são selecionadas de acordo com a particularidade e complexidade do caso, podendo ser empregadas antes, durante ou após a instalação dos implantes.

Além da presença de tecido ceratinizado, outros fatores devem ser levados em consideração para se obter o sucesso dos implantes dentários a longo prazo. O fenótipo gengival, a quantidade de osso periimplantar, o posicionamento e inclinação do implante, bem como as características correspondentes à prótese implantossuportada devem ser considerados na busca de resultados estéticos e funcionais com prognóstico favorável à longo prazo (Bassetti et al., 2017; Chackartchi, Romanos, \& Sculean, 2019).

\section{Resultados e Discussão}

A discussão acerca da necessidade de tecido ceratinizado na região periimplantar já ocorre há décadas e embora alguns estudos iniciais em animais e humanos tenham mostrado que não há correlação entre esse parâmetro e o sucesso dos implantes dentários, (Adell et al., 1986; Bengazi, Wennström, \& Lekholm et al., 1996; Strub, Gaberthüel, \& Grunder et al., 1991) as novas publicações, inclusive com uma série de revisões sistemáticas contestam estes resultados (Brito et al., 2014; Chiu et al., 2015; Gobbato et al., 2013; Lin et al., 2013; Pranskunas et al., 2016).

Isso possivelmente acontece pela própria atualização quanto ao sucesso dos implantes dentários, pois desde o surgimento da implantodontia o parâmetro sucesso passa por alterações, de forma que atualmente se compreende como sucesso a ausência de dor, ausência de mobilidade do implante e ausência de histórico de exsudato periimplantar, bem como perda óssea marginal radiográfica inferior a $2 \mathrm{~mm}$ durante toda a vida (Misch et al., 2008).

Vários estudos nas últimas décadas vêm mostrando que a presença do tecido ceratinizado na região periimplantar apresenta uma série de funções extremamente importante para atingir os parâmetros atuais de sucesso na implantodontia. O tecido ceratinizado proporciona melhor selamento tecidual, protegendo a região do implante de entrada de microrganismos relacionados à periimplantite, além de reagir de forma mais adequada aos traumatismos fisiológicos da mastigação e traumas provenientes da higienização (Adell et al., 1986; Brito et al., 2014; Branemark et al., 1985; Chiu et al., 2015; Gobbato et al., 2013; Lin et al., 2013; Pranskunas et al., 2016).

Além disso, o tecido ceratinizado, principalmente com biotipo espesso, promove proteção, reduz inflamação local, prevenindo recessão gengival, que por sua vez desencadeia resultados estéticos prejudicados, bem como frequentemente associado a uma perda óssea, que uma vez não controlada leva à perda de inserção do implante e consequentemente insucesso do tratamento reabilitador (Adell et al., 1986; Brito et al., 2014; Branemark et al., 1985; Casado et al., 2013; Chiu et al., 2015; Gallucci et al., 2011; Gobbato et al., 2013; Lin et al., 2013; Novaes et al., 2012; Pranskunas et al., 2016).

Por outro lado, Novaes e colaboradores (2012), em revisão de literatura, observaram que a presença da faixa de tecido ceratinizado não é necessária para a sobrevivência dos implantes a longo prazo, isso quando a higiene bucal é realizada de forma adequada, mas enfatizaram que a ausência deste tecido torna a higienização menos confortável para o paciente. 
Embora ainda haja discussão e necessidade de mais estudos para compreensão total dos parâmetros relacionados ao sucesso dos implantes dentários, já é compreendido que a faixa de tecido ceratinizado apresenta papel importante na saúde periimplantar.

Muitas vezes, em virtude do tempo levado entre a perda dentária e a reabilitação implantossuportada, o leito receptor já não dispõe de mucosa ceratinizada no local. Sendo assim, há necessidade de realização de cirurgia para enxertia de mucosa ceratinizada. Quando há presença de mucosa ceratinizada próxima à região de enxertia pode-se optar por enxertos pediculados, enquanto regiões onde não há possibilidade de rotação de retalhos tem necessidade de enxertos livres, sejam eles autógenos ou xenógenos (Bassetti et al., 2017; Luo et al., 2020; Tavelli et al., 2020).

O padrão ouro são os enxertos autógenos, pois apresentam melhores resultados que os enxertos de matriz acelular e os enxertos xenógenos (Bassetti et al., 2017; Luo et al., 2020). Já no que se refere às técnicas para ganho de tecido ceratinizado, A técnica de retalho de espessura parcial reposicionado apicalmente associada ao enxerto gengival livre parece ser a melhor escolha (Luo et al., 2020).

\section{Conclusão}

Diante dos atuais achados da literatura, pode-se observar que uma faixa de mucosa ceratinizada de, pelo menos $2 \mathrm{~mm}$, é necessária para o melhor resultado dos implantes dentários, no entanto outros fatores, tanto de tecidos moles quanto ósseos, devem ser observados para alcançar o sucesso no implantodontia.

Dentro desse contexto, torna-se importante o delineamento de estudos clínicos futuros que comparem, em longo prazo, as respostas obtidas de acordo com a quantidade de mucosa ceratinizada enxertada e o momento ideal para enxertia, considerando as etapas da reabilitação com prótese implantossuportada.

\section{Referências}

Adell, R., Lekholm, U., Rockler, B., Brånemark, P. I., Lindhe, J., Eriksson, B., \& Sbordone, L. (1986). Marginal tissue reactions at osseointegrated titanium fixtures (I). A 3-year longitudinal prospective study. International journal of oral and maxillofacial surgery, 15(1), 39-52. https://doi.org/10.1016/s03009785(86)80010-2

Albrektsson, T., Zarb, G., Worthington, P., \& Eriksson, A. R. (1986). The long-term efficacy of currently used dental implants: a review and proposed criteria of success. The International journal of oral \& maxillofacial implants, 1(1), 11-25.

Bassetti, R. G., Stähli, A., Bassetti, M. A., \& Sculean, A. (2017). Soft tissue augmentation around osseointegrated and uncovered dental implants: a systematic review. Clinical oral investigations, 21(1), 53-70. https://doi.org/10.1007/s00784-016-2007-9

Bengazi, F., Wennström, J. L., \& Lekholm, U. (1996). Recession of the soft tissue margin at oral implants. A 2-year longitudinal prospective study. Clinical oral implants research, 7(4), 303-310. https://doi.org/10.1034/j.1600-0501.1996.070401.x

Bouri, A., Jr, Bissada, N., Al-Zahrani, M. S., Faddoul, F., \& Nouneh, I. (2008). Width of keratinized gingiva and the health status of the supporting tissues around dental implants. The International journal of oral \& maxillofacial implants, 23(2), 323-326.

Brito, C., Tenenbaum, H. C., Wong, B. K., Schmitt, C., \& Nogueira-Filho, G. (2014). Is keratinized mucosa indispensable to maintain peri-implant health? A systematic review of the literature. Journal of biomedical materials research. Part B, Applied biomaterials, 102(3), 643-650. https://doi.org/10.1002/jbm.b.33042

Branemark, P. I., Zarb, G. A., \& Albrektsson, T. (1985). Tissue-integrated prostheses: osseointegration in clinical dentistry. Quintessence.

Carranza, F., Newman, M., Takei, H., \& Klokkevold, P. (2011). Periodontia clínica. Elsevier.

Casado, P., Bonato, L., \& Granjeiro, J. (2013). Relação entre fenótipo periodontal fino e desenvolvimento de doença peri-implantar: avaliação clínicoradiográfica. Braz J Periodontol, 1(23), 68-75.

Chackartchi, T., Romanos, G. E., \& Sculean, A. (2019). Soft tissue-related complications and management around dental implants. Periodontology 2000, 81(1), 124-138. https://doi.org/10.1111/prd.12287

Chiu, Y. W., Lee, S. Y., Lin, Y. C., \& Lai, Y. L. (2015). Significance of the width of keratinized mucosa on peri-implant health. Journal of the Chinese Medical Association: JCMA, 78(7), 389-394. https://doi.org/10.1016/j.jcma.2015.05.001

Dinato, J. C., \& Polido, W. D. (2004). Implantes osseointegrados cirurgia e prótese. Artes Médicas. 
Gallucci, G. O., Grütter, L., Chuang, S. K., \& Belser, U. C. (2011). Dimensional changes of peri-implant soft tissue over 2 years with single-implant crowns in the anterior maxilla. Journal of clinical periodontology, 38(3), 293-299. https://doi.org/10.1111/j.1600-051X.2010.01686.X

Garcia, R. V., Kraehenmann, M. A., Bezerra, F. J., Mendes, C. M., \& Rapp, G. E. (2008). Clinical analysis of the soft tissue integration of non-submerged (ITI) and submerged (3i) implants: a prospective-controlled cohort study. Clinical oral implants research, 19(10), 991-996. https://doi.org/10.1111/j.16000501.2007.01345.x

Gobbato, L., Avila-Ortiz, G., Sohrabi, K., Wang, C. W., \& Karimbux, N. (2013). The effect of keratinized mucosa width on peri-implant health: a systematic review. The International journal of oral \& maxillofacial implants, 28(6), 1536-1545. https://doi.org/10.11607/jomi.3244

Hämmerle, C., \& Tarnow, D. (2018). The etiology of hard- and soft-tissue deficiencies at dental implants: A narrative review. Journal of periodontology, 89 Suppl 1, S291-S303. https://doi.org/10.1002/JPER.16-0810

Heinemann, F., Hasan, I., Bourauel, C., Biffar, R., \& Mundt, T. (2015). Bone stability around dental implants: Treatment related factors. Annals of anatomy = Anatomischer Anzeiger : official organ of the Anatomische Gesellschaft, 199, 3-8. https://doi.org/10.1016/j.aanat.2015.02.004

Javed, F., Ahmed, H. B., Crespi, R., \& Romanos, G. E. (2013). Role of primary stability for successful osseointegration of dental implants: Factors of influence and evaluation. Interventional medicine \& applied science, 5(4), 162-167. https://doi.org/10.1556/IMAS.5.2013.4.3

Kahn, S., Menezes, C. C., Imperial, R. C., Leite, J. S., \& Dias, A. T. (2013). Influência do biótipo periodontal na Implantodontia e na Ortodontia. Rev. bras. odontol., 70(1), 40-45.

Kao, R. T., Fagan, M. C., \& Conte, G. J. (2008). Thick vs. thin gingival biotypes: a key determinant in treatment planning for dental implants. Journal of the California Dental Association, 36(3), 193-198.

Lages, F. S., Douglas-de Oliveira, D. W., \& Costa, F. O. (2018). Relationship between implant stability measurements obtained by insertion torque and resonance frequency analysis: A systematic review. Clinical implant dentistry and related research, 20(1), 26-33. https://doi.org/10.1111/cid.12565

Lin, G. H., Chan, H. L., \& Wang, H. L. (2013). The significance of keratinized mucosa on implant health: a systematic review. Journal of periodontology, 84(12), 1755-1767. https://doi.org/10.1902/jop.2013.120688

Lin, G. H., \& Madi, I. M. (2019). Soft-Tissue Conditions Around Dental Implants: A Literature Review. Implant dentistry, 28(2), 138-143. https://doi.org/10.1097/ID.0000000000000871

Listgarten, M. A., Lang, N. P., Schroeder, H. E., \& Schroeder, A. (1991). Periodontal tissues and their counterparts around endosseous implants [corrected and republished with original paging, article orginally printed in Clin Oral Implants Res 1991 Jan-Mar;2(1):1-19]. Clinical oral implants research, 2(3), 1-19. https://doi.org/10.1034/j.1600-0501.1991.020309.x

Luo, R. M., Chvartszaid, D., Kim, S. W., \& Portnof, J. E. (2020). Soft-Tissue Grafting Solutions. Dental clinics of North America, 64(2), 435-451. https://doi.org/10.1016/j.cden.2019.12.008

Malpartida-Carrillo, V., Tinedo-Lopez, P. L., Guerrero, M. E., Amaya-Pajares, S. P., Özcan, M., \& Rösing, C. K. (2020). Periodontal phenotype: A review of historical and current classifications evaluating different methods and characteristics. Journal of esthetic and restorative dentistry: official publication of the American Academy of Esthetic Dentistry ... [et al.], 10.1111/jerd.12661. Advance online publication. https://doi.org/10.1111/jerd.12661

Marcantonio, C., Nicoli, L. G., Marcantonio Junior, E., \& Zandim-Barcelos, D. L. (2015). Prevalence and Possible Risk Factors of Peri-implantitis: A Concept Review. The journal of contemporary dental practice, 16(9), 750-757. https://doi.org/10.5005/jp-journals-10024-1752

Misch, C. E., Perel, M. L., Wang, H. L., Sammartino, G., Galindo-Moreno, P., Trisi, P., Steigmann, M., Rebaudi, A., Palti, A., Pikos, M. A., Schwartz-Arad, D., Choukroun, J., Gutierrez-Perez, J. L., Marenzi, G., \& Valavanis, D. K. (2008). Implant success, survival, and failure: the International Congress of Oral Implantologists (ICOI) Pisa Consensus Conference. Implant dentistry, 17(1), 5-15. https://doi.org/10.1097/ID.0b013e3181676059

Novaes, V. C. N., Santos, M. R., Almeida, J. M. d., Pellizer, E. P., \& Mendonça, M. R. (2012). A importância da mucosa ceratinizada na implantodontia. Revista Odontológica de Araçatuba, 33(2), 41-46.

Park, J. C., Yang, K. B., Choi, Y., Kim, Y. T., Jung, U. W., Kim, C. S., Cho, K. S., Chai, J. K., Kim, C. K., \& Choi, S. H. (2010). A simple approach to preserve keratinized mucosa around implants using a pre-fabricated implant-retained stent: a report of two cases. Journal of periodontal \& implant science, 40(4), 194-200. https://doi.org/10.5051/jpis.2010.40.4.194

Pranskunas, M., Poskevicius, L., Juodzbalys, G., Kubilius, R., \& Jimbo, R. (2016). Influence of Peri-Implant Soft Tissue Condition and Plaque Accumulation on Peri-Implantitis: a Systematic Review. Journal of oral \& maxillofacial research, 7(3), e2. https://doi.org/10.5037/jomr.2016.7302

Rebollal, J., M., Vidigal-Júnior. G., \& Cardoso, E. S. (2006). Fatores locais que determinam o fenótipo gengival ao redor de implantes dentários: revisão de literatura. ImplantNews, 3(2), 155-160.

Sciasci, P., Casalle, N., \& Vaz, L. G. (2018). Evaluation of primary stability in modified implants: Analysis by resonance frequency and insertion torque. Clinical implant dentistry and related research, 20(3), 274-279. https://doi.org/10.1111/cid.12574

Silva, K. C., Zenóbio, E. G., Souza, P., Soares, R. V., Cosso, M. G., \& Horta, M. (2018). Assessment of Dental Implant Stability in Areas Previously Submitted to Maxillary Sinus Elevation. The Journal of oral implantology, 44(2), 109-113. https://doi.org/10.1563/aaid-joi-D-17-00094

Sorni-Bröker, M., Peñarrocha-Diago, M., \& Peñarrocha-Diago, M. (2009). Factors that influence the position of the peri-implant soft tissues: a review. Medicina oral, patologia oral y cirugia bucal, 14(9), e475-e479.

Strub, J. R., Gaberthüel, T. W., \& Grunder, U. (1991). The role of attached gingiva in the health of peri-implant tissue in dogs. 1. Clinical findings. The International journal of periodontics \& restorative dentistry, 11(4), 317-333. 
Research, Society and Development, v. 10, n. 2, e3510212202, 2021

(CC BY 4.0) | ISSN 2525-3409 | DOI: http://dx.doi.org/10.33448/rsd-v10i2.12202

Tavelli, L., Barootchi, S., Avila-Ortiz, G., Urban, I. A., Giannobile, W. V., \& Wang, H. L. (2021). Peri-implant soft tissue phenotype modification and its impact on peri-implant health: A systematic review and network meta-analysis. Journal of periodontology, 92(1), 21-44. https://doi.org/10.1002/JPER.190716

Zigdon, H., \& Machtei, E. E. (2008). The dimensions of keratinized mucosa around implants affect clinical and immunological parameters. Clinical oral implants research, 19(4), 387-392. https://doi.org/10.1111/j.1600-0501.2007.01492.x

Zita Gomes, R., de Vasconcelos, M. R., Lopes Guerra, I. M., de Almeida, R., \& de Campos Felino, A. C. (2017). Implant Stability in the Posterior Maxilla: A Controlled Clinical Trial. BioMed research international, 2017, 6825213. https://doi.org/10.1155/2017/6825213 